\section{Impact of CRAB symptoms in survival of patients with symptomatic myeloma in novel agent era}

\author{
Aya Nakaya, Shinya Fujita, \\ Atsushi Satake, Takahisa Nakanishi, \\ Yoshiko Azuma, Yukie Tsubokura, \\ Masaaki Hotta, Hideaki Yoshimura, \\ Kazuyoshi Ishii, Tomoki Ito, \\ Shosaku Nomura
}

First Department of Internal Medicine, Kansai Medical University, Hirakata, Japan

\begin{abstract}
The acronym $C R A B$ summarizes the most typical clinical manifestations of multiple myeloma, these being hypercalcemia, renal failure, anemia, and bone disease. CRAB can be used to distinguish between active, symptomatic multiple myeloma and monoclonal gammopathy of undermined significance or smoldering myeloma. The distinction is relevant not only for classification and diagnosis but also for therapy. CRAB factors influence the prognosis of multiple myeloma. However, it is unclear whether the presence of CRAB factors has an influence on the prognosis of myeloma treated with novel agents. In the current study, patients with hypercalcemia and bone disease showed a significantly worse prognosis, whereas anemia and renal failure showed no difference in survival. Novel agents used for treatment of patients with renal failure suggested a favorable outcome compared with conventional therapy. Bone disease was the most common factor and may have the strongest prognostic value in symptomatic myeloma patients using novel agents.
\end{abstract}

\section{Introduction}

The acronym $C R A B$ was defined in criteria of the International Myeloma Working Group in 2003 to simplify the most typical clinical manifestations of multiple myeloma, these being hypercalcemia, renal failure, anemia, and bone disease. ${ }^{1}$ CRAB factors can be used to distinguish between active, symptomatic multiple myeloma and its precursor states, monoclonal gammopathy of undermined significance and smoldering myeloma. The distinction is relevant not only for classification and diagnosis, but also for therapy. CRAB factors are criteria for deciding when to initiate chemotherapy and are known to suggest the prognosis of multiple myeloma. The staging criteria developed by Durie and Salmon have made use of all CRAB clinical features, because serum calcium, creatinine, and hemoglobin levels, and the number of bone lesions are essential in defining disease stage. ${ }^{2}$

With advances in technology and science, it is unclear whether the presence of CRAB factors is associated with the prognosis of myeloma treated with novel agents. With regard to renal failure, there are several reports that suggest that novel agents have improved renal dysfunction, yet survival has not improved. ${ }^{3-7}$ For CRAB factors, little is known about their relationship with survival.

In the current study, we examined the presence of CRAB factors on survival in symptomatic myeloma patients treated with novel agents.

\section{Materials and methods}

\section{Patients and treatments}

A total of 113 consecutively diagnosed symptomatic myeloma patients at Kansai Medical University Hospital between 2006 and 2014 were included in this retrospective analysis. Hypercalcemia was defined as a serum calcium level $>11.0 \mathrm{mg} / \mathrm{dL}$, anemia as a hemoglobin level $<10.0 \mathrm{~g} / \mathrm{dL}$, renal failure as estimated glomerular filtration rate $<40 \mathrm{~mL} / \mathrm{min}$, and bone lesion as one or more osteolytic lesions on skeletal radiography, computed tomography, ${ }^{18} \mathrm{~F}$-fluorodexyglucose positron emission tomography with computed tomography, or magnetic resonance imaging following new International Myeloma Working Group criteria. ${ }^{8}$ The novel agents were thalidomide, bortezomib, and lenalidomide. Conventional therapies are traditional cytotoxic agents, these being alkylators, anthracyclines, Vinca alkaloids, and steroids. Patients treated with novel agents included those who started with conventional therapies and switched to novel agents. The conventional therapy group was treated only with conventional therapies and with no novel agents.

\section{Statistical analysis}

Overall survival was calculated from diagnosis until the time of death or the last clinical follow-up and was analyzed using the Kaplan-Meier method. Results were compared using log-rank statistics and Cox proportional hazards models. A P-value
Correspondence: Aya Nakaya, First Department of Internal Medicine, Kansai Medical University, 2-5-1, Shin-machi, Hirakata, Osaka 573-1010, Japan.

Tel.: +81.72.804.2503.

E-mail:nakaya1016@yahoo.co.jp

Key words: CRAB; symptomatic myeloma; novel agent; conventional therapy.

Contributions: the authors contributed equally.

Conflict of interest: the authors declare no potential conflict of interest.

Received for publication: 14 September 2016. Accepted for publication: 28 December 2016.

This work is licensed under a Creative Commons Attribution-NonCommercial 4.0 International License (CC BY-NC 4.0).

CCopyright A. Nakaya et al., 2017

Licensee PAGEPress, Italy

Hematology Reports 2017; 9:6887

doi:10.4081/hr.2017.6887

$<0.05$ was considered statistically significant. All statistical analyses were performed using EZR (modified R software; Saitama Medical Center, Jichi Medical University, Saitama, Japan). This retrospective analysis was approved by the local ethics committee of Kansai Medical University.

\section{Results}

\section{Patient characteristics}

We retrospectively analyzed 113 symptomatic myeloma patients diagnosed between January 2006 and December 2014 at Kansai Medical University Hospital. Table 1 summarizes patient characteristics at the time of diagnosis. Mean age was 72 years old (range: $34-88$ years) and 51\% were male. $M$ protein comprised $\mathrm{IgG}$ $(69 \%), \operatorname{IgA}(14 \%)$, and Bence Jones protein (14\%). According to the International Scoring System, stage 1 was seen in $45 \%$ of patients, stage 2 in $21 \%$, and stage 3 in $34 \%$. Forty-one percent of patients were treated with conventional therapies, and $59 \%$ were treated with novel agents. Among them, $13 \%$ of patients were treated with high-dose chemotherapy followed by autologous stem cell transplantation.

\section{Frequency of CRAB}

The frequency of CRAB factors is summarized in Table 1. The most commonly 
presenting factor of multiple myeloma was bone disease $(68 \%)$, followed by anemia $(57 \%)$, renal failure $(29 \%)$, and hypercalcemia $(6 \%)$.

\section{Overall survival according to CRAB}

The presence of factors and patient outcomes with symptomatic multiple myeloma were compared (Figure 1). Patients with hypercalcemia and bone disease showed significantly worse survival (Figure 1A,D), whereas those patients with anemia or renal failure showed no difference in survival (Figure 1B,C).

\section{Overall survival according to therapy}

We investigated whether novel agents were able to alleviate CRAB factors. Patients were divided into two groups according to therapy: novel agents or conventional therapies. Renal failure treated with novel agents showed a tendency of a favorable outcome (median overall survival: 101 months) compared with conventional therapy (median overall survival: 42 months), although the finding was not statistically significant $(\mathrm{P}=0.686)$ (Figure 2B). Anemia and bone disease both tended to show that novel agents did not surpass conventional therapies, although the findings were not statistically significant $(\mathrm{P}=0.567$ and $\mathrm{P}=0.76$, respectively) (Figure $2 \mathrm{C}, \mathrm{D})$. There were too few cases of hypercalcemia to estimate the influence of therapy (Figure 2A).

Table 1. Patients' characteristics.

\begin{tabular}{lc} 
Characteristic & N. \\
N. of patients & 113 \\
Median age, range & $72(34-88)$ \\
\hline Male (\%) & 51 \\
M protein (\%) & \\
IgG & 69 \\
IgA & 14 \\
Bence Johns protein & 14 \\
Others & 3 \\
\hline ISS (\%) & \\
I & 45 \\
II & 21 \\
III & 34 \\
Theraphy (\%) & \\
Conventional & 41 \\
Novel & 59 \\
Autologous stem cell transplantation & 13 \\
\hline Frequecny of CRAB factors (\%) & \\
$\quad$ Hypercalcemia & 6 \\
Renal Failure & 29 \\
Anemia & 57 \\
Bone disease & 68 \\
Median follow up, months & $37(1.5-110)$ \\
\hline
\end{tabular}

A

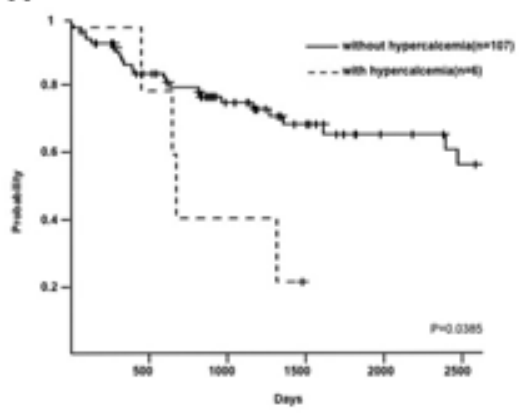

C

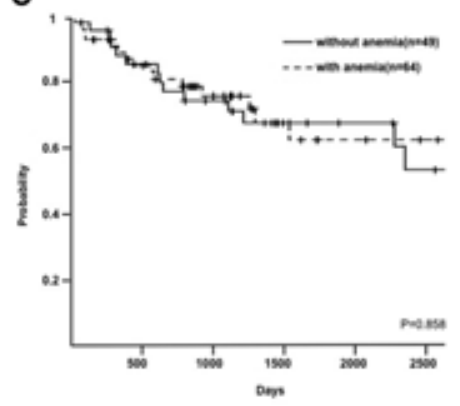

B

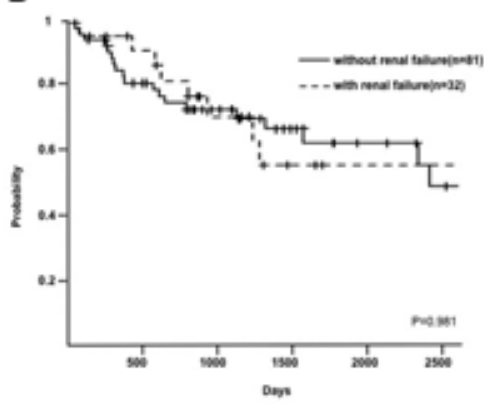

D

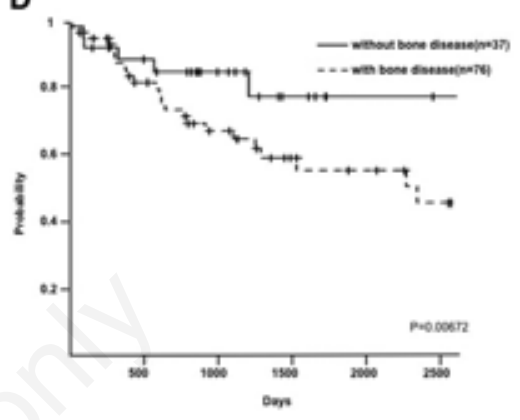

Figure 1. Survival comparison between with CRAB vs. without CRAB factors. A) With hypercalcemia or without hypercalcemia. Median overall survival was 32 months $v s$. 101 months, respectively $(P=\mathbf{0 . 0 3 8 5})$. B) With renal failure or without renal failure. Median overall survival was 101 months vs. 96 months, respectively $(P=0.981)$. C) With anemia or without anemia. Median overall survival was 101 months vs. 96 months, respectively $(P=0.858)$. $D)$ With bone disease or without bone disease. Median overall survival was 80 months $v s$. data not available, respectively $(\mathrm{P}=0.00672)$.

A

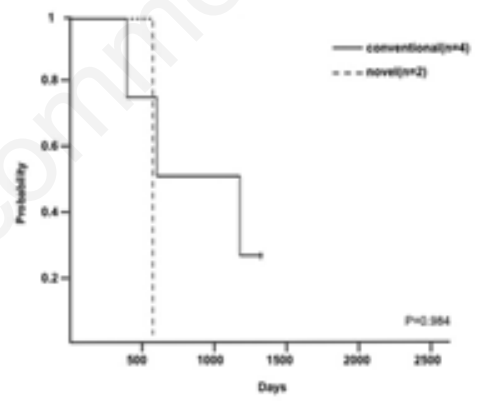

C

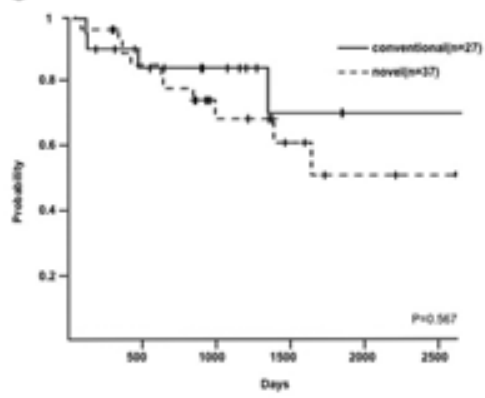

B

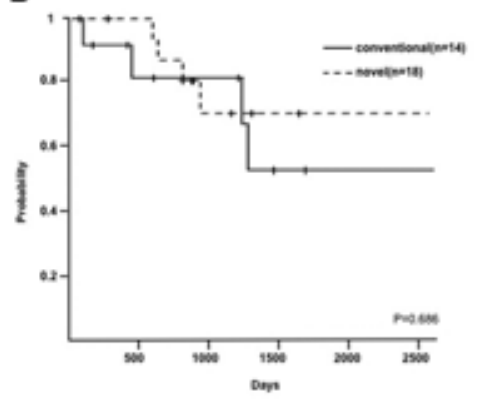

D

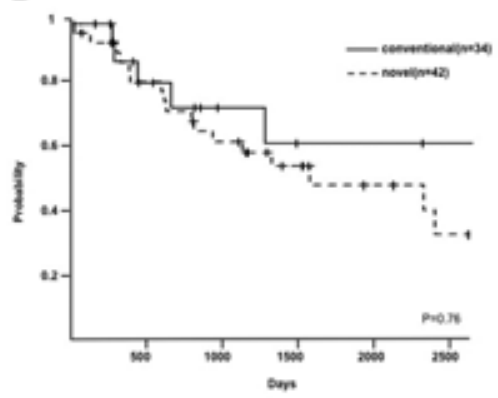

Figure 2. Overall survival according to therapy. A) With hypercalcemia, median overall survival of conventional therapy was 32 months, and with novel agents it was 20 months $(P=0.984)$. B) With renal failure, median overall survival of conventional therapy was 42 months, and with novel agents it was 101 months $(P=0.686)$. C) With anemia, median overall survival of conventional therapy was 105 months, and with novel agents it was 101 months $(P=0.567)$. D) With bone disease, median overall survival of conventional therapy was 96 months, and with novel agents it was 78 months $(P=0.76)$. 


\section{Discussion and Conclusions}

This study assessed CRAB factors and their association with symptomatic myeloma patients' prognoses following treatment with novel agents. Patients with hypercalcemia and bone disease showed a significantly worse prognosis, whereas anemia and renal failure showed no difference in survival. Novel agents used to treat patients with renal failure appeared to have a favorable outcome compared with conventional therapies, suggesting that renal failure might have been overcome by novel agents. Previous studies revealed that treatment with novel agents improved renal dysfunction, although improvement of renal dysfunction leading to improved survival in patients with renal failure has not been proven. ${ }^{3-7}$ The results of the current study suggest the possibility that novel treatment agents might have improved the survival of patients with renal failure.

Bone disease tends to be the most common factor and may show the strongest prognostic value, reflecting tumor burden and indicating that the disease is close to the end stage. However, the use of novel agents did not show any improvement in the prognosis of patients with bone disease in the current study. This suggests that a greater tumor burden cannot be treated to the level required to successfully prolong survival, even with novel agents.

Anemia can occur from both myeloma and renal failure, therefore anemia may not reflect tumor burden directly. The currently used staging system for myeloma, the International Scoring System, does not include hemoglobin as an evaluation item. Nowadays, CRAB factors are criteria to help determine the initiation of chemotherapy; however, anemia does not reflect prognosis. It is therefore vital that when anemia is used to help determine the initiation of chemotherapy, the patient's condition is carefully assessed.

The presence of hypercalcemia suggests a poor prognosis. However, there were very few patients with hypercalcemia in the current study. Therefore, the influence of hypercalcemia on the prognosis of patients treated with novel agents could not be accurately determined.

This study had several limitations. First, it was a retrospective and single institution study with a small number of patients. Second, it would have been beneficial to have investigated isolated symptoms to determine an accurate prognosis. That said, however, there were few patients with a single symptom, most patients having multiple (overlapping) symptoms. We counted the number of symptoms in each patient even when they overlapped. Finally, although genetic and biological backgrounds may have an influence on outcome, we did not conduct chromosome testing in detail because of limitations presented by possible insurance claims.

Despite these limitations, our findings suggest that bone disease may have the strongest prognostic value, renal failure might have been overcome by novel agents, and anemia might no longer be a reliable predictive factor.

In conclusion, among CRAB factors, the presence of bone disease appeared to be the most useful to predict the prognosis of symptomatic myeloma patients treated with novel agents.

\section{References}

1. Durie BG, Salmon SE. A clinical staging system for multiple myeloma. Correlation of measured myeloma cell mass with presenting clinical features, response to treatment, and survival.
Cancer 1975;36:842-54

2 International Myeloma Working Group. Criteria for the classification of monoclonal gammopathies, multiple myeloma and related disorders: a report of the International Myeloma Working Group. Br J Haematol 2003;121:749-57.

3. Dimopoulos MA, Richardson PG, Schlag R, et al. VMP (Bortezomib, Melphalan, and Prednisone) is active and well tolerated in newly diagnosed patients with multiple myeloma with moderately impaired renal function, and results in reversal of renal impairment: cohort analysis of the phase III VISTA study. J Clin Oncol 2009;27:6086-93.

4. Ludwig H, Adam Z, Hajek R, et al. Light chain-induced acute renal failure can be reversed by bortezomib-doxorubicin-dexamethasone in multiple myeloma: results of a phase II study. J Clin Oncol 2010;28:4635-41.

5. Chanan-Khan AA, Kaufman JL, Mehta $\mathrm{J}$, et al. Activity and safety of bortezomib in multiple myeloma patients with advanced renal failure: a multicenter retrospective study. Blood 2007;109:2604-6.

6. Roussou M, Kastritis E, Christoulas D, et al. Reversibility of renal failure in newly diagnosed patients with multiple myeloma and the role of novel agents. Leuk Res 2010;34:1395-7.

7. Dimopoulos MA, Roussou M, Gkotzamanidou $\mathrm{M}$, et al. The role of novel agents on the reversibility of renal impairment in newly diagnosed symptomatic patients with multiple myeloma. Leukemia 2013;27:423-9.

8. Rajkumar SV, Dimopoulos MA, Palumbo A, et al. International Myeloma Working Group updated criteria for the diagnosis of multiple myeloma. Lancet Oncol 2014;15:e53848. 